\title{
Wronskian-based tests for stability of polynomial combinations
}

\author{
Christophe FONTE, Cédric DELATTRE \\ Centre de Recherche en Automatique de Nancy (CRAN),UMR-7039, France \\ Nancy-Université, CNRS. \\ Christophe.Fontedcran.uhp-nancy. fr \\ Cedric.Delattrediut-longwy uhp-nancy. fr
}

\begin{abstract}
In this paper, a stability criterion based on counting the real roots of two specific polynomials is formulated. To establish this result, it is shown that a hyperbolicity condition and a strict positivity of a polynomial Wronskian are necessary and sufficient for the stability of any real polynomial. This result is extended to the stability study of some linear combinations of polynomials. Necessary and sufficient conditions of stability are obtained for polynomial segments and planes.
\end{abstract}

Keywords: hyperbolic polynomial, Hurwitz polynomial, Hermite-Biehler theorem, uncertain polynomial, Wronskian, Kharitonov polynomials.

\footnotetext{
Notations

$\mathbb{R}^{n}$ denotes the set of real vectors of dimension $n$.

$\mathbb{P}_{m}$ denotes the set of real polynomials of degree $m$.

$\mathbb{H}_{m}$ is the set of hyperbolic polynomials of degree $m$.

$W(g, h)(s)$ with $s \in I$ denotes the Wronskian of the two functions $g(s)$ and $h(s)$ differentiable on the interval

$\mathfrak{W}(g, h)(s)$ with $s \in I$ denotes the Wronskian of the two functions $g\left(-s^{2}\right)$ and $s h\left(-s^{2}\right)$ differentiable on the interval $I$.
} $I$.

\section{INTRODUCTION}

A real polynomial is said to be stable (or Hurwitz) if all its roots lie in the open left half of the complex plane. This paper studies stability of polynomial combinations, particularly questions regarding stability of polynomial segments and polynomial planes.

For developing our approach, preliminary results are given in section 2. For that, we use an alternative to the Hermite-Biehler theorem without explicitly considering the zeroes interlacing property. This property is replaced by a double condition constituted on one hand by a polynomial positivity condition and on the other hand by a hyperbolicity condition (Recall that a polynomial is called hyperbolic if all its roots are real). Thereafter, this double requirement is reduced to a test for counting the real roots of polynomials by a discriminant method, see [10] and [15]. Then a link between Hermite's criterion and this stability method is established. 
Our framework permits to extend this issue on stability of one polynomial to stability of polynomial segments and planes.

In the first part of section 3, the convex polynomial segments are considered. We can note that the stability of convex segments with invariant degree has been examined by numerous authors, see [4], [5], [3], [6] and [2]. For example [4] was among the first to give a necessary and sufficient condition equivalent to eigenvalues negativity condition carrying on a product of matrices under the assumption that the endpoints are Hurwitz stable. Others approaches use methods based on the zero exclusion principle, see [2] or the stability boundary theorem, see [6] or even the strong stabilization, see [5]. To solve this problem, we propose a new alternative that consists to apply our previous result on the stability of one polynomial to the polynomial segments. We show that a convex polynomial segment is stable under hyperbolicity assumptions if and only if a weak zeroes interlacing property and polynomial positivity conditions are satisfied.

In the second part of section 3, a generalization of these results are provided for different polynomial planes. Necessary and sufficient conditions are obtained for the Kharitonov's plane that complete these given in [7]. These results show that the stability of the Kharitonov's plane depends on a weak zeroes interlacing property and polynomial positivity conditions in connection with the four Kharitonov's polynomials. Then this approach is expanded to any polynomial plane. In this case, necessary and sufficient conditions are reduced to test some relations of polynomial positivity under assumptions of hyperbolicity and of zeroes interlacing property.

\section{Polynomial stability}

In this section, a criterion of polynomial stability is established which is based on the counting of the real roots of two polynomials. As a preliminary result, a link is made between the polynomial stability and the sign of a polynomial Wronskian by using the roots interlacing property and the Hermite-Biehler theorem.

\section{A. Hermite-Biehler stability}

Definition 1: [10], Zeroes Interlacing Property.

Let $P(u)$ and $Q(u)$ be two real polynomials of respective degrees $l$ and $l-1$ (or $l$ ). Let us assume the roots of these polynomials defined by the following sets

$$
\begin{aligned}
\operatorname{root}(P(u)) & =\left\{u_{1}, \ldots, u_{l}\right\} \\
\operatorname{root}(Q(u)) & =\left\{v_{1}, \cdots, v_{l-1}\right\} \\
(\text { or } \operatorname{root}(Q(u)) & \left.=\left\{v_{1}, \cdots, v_{l}\right\}\right)
\end{aligned}
$$

Then $P(u)$ and $Q(u)$ interlace iff

- the leading coefficients of $P(u)$ and $Q(u)$ have the same sign,

- the roots of $P(u)$ and $Q(u)$ are real, simple and distinct,

- the $l$ roots of $P(u)$ alternate with the $l-1$ (or $l$ ) roots of $Q(u)$ as follows

$$
\begin{aligned}
& \quad u_{1}<v_{1}<u_{2}<v_{2} \ldots<u_{l-1}<v_{l-1}<u_{l} \\
& \left(\text { or } v_{1}<u_{1}<v_{2} \ldots<u_{l-1}<v_{l}<u_{l}\right)
\end{aligned}
$$


Let $f$ be in $\mathbb{P}_{m}$ given by

$$
f(s)=a_{m} s^{m}+a_{m-1} s^{m-1} \cdots+a_{2} s^{2}+a_{1} s+a_{0}
$$

Let $f$ be expanded as (2) where $f^{e}$ and $f^{o}$ denote the even part and the odd part of $f$ respectively.

$$
f(s)=f^{e}\left(s^{2}\right)+s f^{o}\left(s^{2}\right)
$$

The relationship between Zeroes Interlacing Property and Hurwitz stability is emphasized by the well known Hermite-Biehler Theorem that is recalled below.

Theorem 1: E.g. [10], Hermite-Biehler's Theorem.

The real polynomial $f$ is stable iff $f^{e}\left(-s^{2}\right)$ and $s f^{o}\left(-s^{2}\right)$ satisfy the Zeroes Interlacing Property.

The next result arises by considering Theorem 1. This result proposes to use a polynomial Wronskian for testing polynomial stability.

Theorem 2: Let $f$ be in $\mathbb{P}_{m}$. The polynomial $f$ is stable iff the following two conditions hold

a) the roots of $f^{e}\left(-s^{2}\right)$ are real,

b) $\forall s \in \mathbb{R}$,

$$
\mathfrak{W}\left(f^{e}, f^{o}\right)(s)=\left|\begin{array}{ll}
f^{e}\left(-s^{2}\right) & s f^{o}\left(-s^{2}\right) \\
\frac{d f^{e}\left(-s^{2}\right)}{d s} & \frac{d\left(s f^{o}\left(-s^{2}\right)\right)}{d s}
\end{array}\right|>0
$$

\section{Proof: See Appendix}

B. Counting the number of distinct real roots of a polynomial.

In order to make this paper self-contained and to give a practical significance of algebraic conditions a) and b) given in Theorem 2, an explicit criterion to count the number of real roots of polynomials $f^{e}\left(-s^{2}\right)$ and $\mathfrak{W}\left(f^{e}, f^{o}\right)(s)$ is produced in this subsection.

It is known that the discriminant of a polynomial is an expression which provides information about the nature of its roots. That is why a formulation based on the discrimination matrix of a real polynomial is hereafter given, see [10],[15],[14].

Consider a polynomial $g(s)$ in $\mathbb{P}_{m}$

$$
g(s)=\sigma_{m} s^{m}+\sigma_{m-1} s^{m-1} \cdots+\sigma_{2} s^{2}+\sigma_{1} s+\sigma_{0}
$$

Let $R\left(g, g^{\prime}\right)$ be the $2 m \times 2 m$ Sylvester matrix given below

$$
\left[\begin{array}{llllllll}
\sigma_{m} & \sigma_{m-1} & \sigma_{m-2} & \ldots & \sigma_{0} & & & \\
0 & m \sigma_{m} & (m-1) \sigma_{m-1} & \ldots & \sigma_{1} & & \\
0 & 0 & \sigma_{m} & \ldots & \sigma_{1} & \sigma_{0} & & \\
\vdots & & & & & & & \vdots \\
& & & \sigma_{m} & \sigma_{m-1} & \ldots & \sigma_{1} & \sigma_{0} \\
& & & 0 & m \sigma_{m} & (m-1) \sigma_{m-1} & \ldots & \sigma_{1}
\end{array}\right]
$$


The even order minors of the matrix $R\left(g, g^{\prime}\right)$ permit to determine the number of distinct (real and complex) roots of $g$. Denote by $D_{k}$ the determinant of the submatrix formed by the first $k$ rows and the first $k$ columns of $R\left(g, g^{\prime}\right)$ for $k=1,2 \ldots, m$.

Definition 2: The discriminant sequence $D$.

The $m$-tuple $D=\left[D_{1}, D_{2} \ldots, D_{m}\right]$ is called the discriminant sequence of $g$.

Definition 3: The sign list $\Psi(D)$.

Let $D=\left[D_{1}, D_{2} \ldots, D_{m}\right]$ be the discriminant sequence of $g$. We denote by $\Psi(D)=\left[\operatorname{sign}\left(D_{1}\right), \operatorname{sign}\left(D_{2}\right) \ldots, \operatorname{sign}\left(D_{m}\right)\right]$, the sign list of $D$ where $\operatorname{sign}($.$) is the sign$ function namely

$$
\operatorname{sign}\left(D_{i}\right)= \begin{cases}1 & \text { if } D_{i}>0 \\ 0 & \text { if } D_{i}=0 \\ -1 & \text { if } D_{i}<0\end{cases}
$$

Definition 4: The revised sign list $\Pi(D)$.

Let $\left[\operatorname{sign}\left(D_{i}\right), \operatorname{sign}\left(D_{i+1}\right), \ldots \operatorname{sign}\left(D_{i+j}\right)\right]$, a section of the sign list $\Psi(D)$ where $\operatorname{sign}\left(D_{i}\right) \neq$ $0, \operatorname{sign}\left(D_{i+1}\right)=\operatorname{sign}\left(D_{i+2}\right)=\ldots=\operatorname{sign}\left(D_{i+j-1}\right)=0, \operatorname{sign}\left(D_{i+j}\right) \neq 0$. The revised sign list is get by substituting in the sign list $\Psi(D)$ the subsection $\left[\operatorname{sign}\left(D_{i+1}\right), \ldots . \operatorname{sign}\left(D_{i+j-1}\right)\right]$ by

$$
\left[-\operatorname{sign}\left(D_{i}\right),-\operatorname{sign}\left(D_{i}\right), \operatorname{sign}\left(D_{i}\right), \operatorname{sign}\left(D_{i}\right),-\operatorname{sign}\left(D_{i}\right),-\operatorname{sign}\left(D_{i}\right), \ldots\right]
$$

Recall a result for counting the number of distinct real roots of a polynomial, see [15],[14].

Lemma 1: [15],[14] The number of distinct real roots of $g$ is equal to $\mu-2 \gamma$ where $\mu$ and $\gamma$ are respectively the number of nonzero elements and the the number of sign changes in the revised sign list $\Pi(D)$.

From this Lemma 1 and Theorem 2, a test is deduced for deciding of the polynomial stability.

Corollary 1: The real polynomial $f$ in $\mathbb{P}_{m}$ is stable iff all following conditions hold

a) The revised sign list of the discriminant sequence of $f^{e}\left(-s^{2}\right)$ has no zero element and has no sign change,

b) $\mathfrak{W}\left(f^{e}, f^{o}\right)(s)$ has no real root, i.e. the revised sign list of the discriminant sequence of $\mathfrak{W}\left(f^{e}, f^{o}\right)(s)$ has twice as many nonzero elements as sign changes,

c) $\mathfrak{W}\left(f^{e}, f^{o}\right)\left(s_{0}\right)>0$ for some real $s_{0}$.

Proof: Obvious, see Theorem 2. Concerning conditions b) and c), we know that an univariate polynomial is strictly positive if this polynomial has no real roots and if it is strictly positive for some real value. 


\section{Connection between Hermite's criterion and Wronskian's condition.}

A known result for the polynomial stability is the Hermite's criterion, see e.g. [13]. In this part, we examine the relationships between Hermite's criterion and the Wronskian's condition (3) of Theorem 2.

Theorem 3: (Hermite). A real polynomial $f$ in $\mathbb{P}_{m}$ is stable iff the $m \times m$ symmetric matrix $B=\left[b_{i j}\right]$ is positive definite, where the entries $b_{i j}$ are defined by

$$
\begin{aligned}
& \forall\left(s_{1}, s_{2}\right) \in \mathbb{R}^{2}, \\
& \sum_{i=1}^{m} \sum_{j=1}^{m} b_{i j} s_{1}^{i-1} s_{2}^{j-1}=\frac{s_{1} f^{o}\left(-s_{1}^{2}\right) f^{e}\left(-s_{2}^{2}\right)-f^{e}\left(-s_{1}^{2}\right) s_{2} f^{o}\left(-s_{2}^{2}\right)}{s_{1}-s_{2}}
\end{aligned}
$$

Rewrite right term of relation (4) as it follows

$$
f^{e}\left(-s_{1}^{2}\right) \frac{s_{1} f^{o}\left(-s_{1}^{2}\right)-s_{2} f^{o}\left(-s_{2}^{2}\right)}{s_{1}-s_{2}}-s_{1} f^{o}\left(-s_{1}^{2}\right) \frac{f^{e}\left(-s_{1}^{2}\right)-f^{e}\left(-s_{2}^{2}\right)}{s_{1}-s_{2}}
$$

Considering $s_{1}=s$ and $s_{2}=s+\epsilon$, and computing limit of expression (5) as $\epsilon$ approaches 0 leads to $\mathfrak{W}\left(f^{e}, f^{o}\right)(s)$. From Hermite's criterion, $f$ is stable iff (4) is a positive form. Then we have

$$
\forall s \in \mathbb{R}, \quad \mathfrak{W}\left(f^{e}, f^{o}\right)(s)=\sum_{i=1}^{m} \sum_{j=1}^{m} b_{i j} s^{i+j-2}=V^{T}(s) B V(s)>0
$$

where $V^{T}(s)=\left[\begin{array}{lll}1 & s & s^{2} \ldots s^{m-1}\end{array}\right]$. Note that condition b) in Theorem 2 is not equivalent to the definite-positivity of the matrix $B$ given by expression (4). Indeed $B$ is definite positive iff $x^{T} B x>0$ for any $x \in \mathbb{R}^{m}$ while the Wronskian in equation (3) is positive iff $V^{T} B V>0$ for any $V$ Vandermonde vector of $\mathbb{R}^{m}$. We prove through the below example that condition a) of Theorem 2 is necessary.

Example. Consider the following unstable polynomial $f(s)=(1-s)(s+1)^{2}$. We get

$$
s f^{o}\left(-s^{2}\right)=s^{3}+s, \quad f^{e}\left(-s^{2}\right)=s^{2}+1
$$

This yields to

$$
\forall s \in \mathbb{R}, \mathfrak{W}\left(f^{e}, f^{o}\right)(s)=\left(s^{2}+1\right)^{2}>0
$$

Moreover we have

$$
B=\left(\begin{array}{ll}
1 & 1 \\
1 & 1
\end{array}\right)
$$

Then $B$ is not definite positive. This is in accordance with the fact that $f^{e}\left(-s^{2}\right)$ has no real root although $\mathfrak{W}\left(f^{e}, f^{o}\right)(s)$ is positive. 


\section{Stability OF SOME POLYNOMial COMbinations}

Theorem 2 establishes a stability test for an unique polynomial. This result is extended to polynomial sets.

\section{A. Stability of polynomial segments}

Firstly, we consider the issue of polynomial stability of segments of the form $\alpha f_{0}+(1-\alpha) f_{1}$ with $\alpha \in[0,1]$ without assuming the stability of the two endpoints of same degree $f_{0}$ and $f_{1}$. For that, a preliminary result is derived from Theorem 2.1 given in [8], [9].

Lemma 2: [8], [9] Let $f_{0}^{e}\left(-s^{2}\right)$ and $f_{1}^{e}\left(-s^{2}\right)$, be two polynomials in $\mathbb{H}_{m}$ with leading coefficients of same sign and with roots given by the sets below

$$
\begin{aligned}
& \operatorname{root}\left(f_{0}^{e}\left(-s^{2}\right)\right)=\left\{s_{0}^{e_{0}}, \ldots, s_{m}^{e_{0}}\right\} \\
& \operatorname{root}\left(f_{1}^{e}\left(-s^{2}\right)\right)=\left\{s_{0}^{e_{1}}, \cdots, s_{m}^{e_{1}}\right\}
\end{aligned}
$$

In these conditions, the two following statements are equivalent

a) for each $\alpha \in[0,1]$ the roots of $f_{\alpha}^{e}\left(-s^{2}\right)$ in (8) are real

$$
f_{\alpha}^{e}\left(-s^{2}\right)=\alpha f_{0}^{e}\left(-s^{2}\right)+(1-\alpha) f_{1}^{e}\left(-s^{2}\right)
$$

b) for each $k=1, \ldots, m-1$, we have

$$
\max \left(s_{k}^{e_{0}}, s_{k}^{e_{1}}\right) \leq \min \left(s_{k+1}^{e_{0}}, s_{k+1}^{e_{1}}\right)
$$

Proof: In [8], [9], the proof of Theorem 2.1 assumes that the two endpoints of (8) are two monic polynomials. This proof still holds if we consider as assumption that $f_{0}^{e}\left(-s^{2}\right)$ and $f_{1}^{e}\left(-s^{2}\right)$ have leading coefficients of same sign.

Note that (9) is a weaker condition than the Zeroes Interlacing Property, see Definition 1.

Theorem 4: Consider the polynomial segment $f_{\alpha}$ given by (10)

$$
\forall \alpha \in[0,1], \quad f_{\alpha}(s)=\alpha f_{0}(s)+(1-\alpha) f_{1}(s)
$$

where $f_{0}$ and $f_{1}$ are two polynomials in $\mathbb{P}_{m}$.

Denote by $f_{0}^{e}$ and $f_{1}^{e}$ respectively the even parts of $f_{0}$ and $f_{1}$ that satisfy the following properties

- the leading coefficients of $f_{0}^{e}\left(-s^{2}\right)$ and $f_{1}^{e}\left(-s^{2}\right)$ have the same sign,

- the roots of $f_{0}^{e}\left(-s^{2}\right)$ and $f_{1}^{e}\left(-s^{2}\right)$ given by the sets (6) and (7) are real.

Then the polynomial segment $f_{\alpha}$ is stable iff the inequalities (11) hold.

$$
\left\{\begin{array}{c}
\forall s \in \mathbb{R}, \quad \mathfrak{W}\left(f_{0}^{e}, f_{0}^{o}\right)(s)>0, \quad \mathfrak{W}\left(f_{1}^{e}, f_{1}^{o}\right)(s)>0, \\
\sqrt{\mathfrak{W}\left(f_{0}^{e}, f_{0}^{o}\right)(s) \mathfrak{W}\left(f_{1}^{e}, f_{1}^{o}\right)(s)}+\frac{1}{2}\left(\mathfrak{W}\left(f_{0}^{e}, f_{1}^{o}\right)(s)+\mathfrak{W}\left(f_{1}^{e}, f_{0}^{o}\right)(s)\right)>0 \\
\forall k=1, \ldots, m-1, \quad \max \left(s_{k}^{e_{0}}, s_{k}^{e_{1}}\right) \leq \min \left(s_{k+1}^{e_{0}}, s_{k+1}^{e_{1}}\right)
\end{array}\right.
$$


Proof: As previously defined for $f$ in (2), denote by $f_{\alpha}^{e}$ and $f_{\alpha}^{o}$, the even part and the odd part respectively of the polynomial segment $f_{\alpha}$. Then we have

$$
f_{\alpha}(s)=f_{\alpha}^{e}\left(s^{2}\right)+s f_{\alpha}^{o}\left(s^{2}\right)
$$

where $f_{\alpha}^{e}\left(s^{2}\right)$ and $s f_{\alpha}^{o}\left(s^{2}\right)$ are given by the following expressions

$$
\left\{\begin{array}{l}
f_{\alpha}^{e}\left(s^{2}\right)=\alpha f_{0}^{e}\left(s^{2}\right)+(1-\alpha) f_{1}^{e}\left(s^{2}\right) \\
f_{\alpha}^{o}\left(s^{2}\right)=\alpha f_{0}^{o}\left(s^{2}\right)+(1-\alpha) f_{1}^{o}\left(s^{2}\right)
\end{array}\right.
$$

and where $s f_{0}^{o}\left(s^{2}\right)$ and $s f_{1}^{o}\left(s^{2}\right)$ are the odd parts of $f_{0}(s)$ and $f_{1}(s)$, respectively. Furthermore, we have

$$
\begin{aligned}
& \mathfrak{W}\left(f_{\alpha}^{e}, f_{\alpha}^{o}\right)(s)=\alpha^{2} \mathfrak{W}\left(f_{0}^{e}, f_{0}^{o}\right)(s)+\alpha(1-\alpha)\left[\mathfrak{W}\left(f_{0}^{e}, f_{1}^{o}\right)(s)+\mathfrak{W}\left(f_{1}^{e}, f_{0}^{o}\right)(s)\right]+ \\
& \quad(1-\alpha)^{2} \mathfrak{W}\left(f_{1}^{e}, f_{1}^{o}\right)(s)
\end{aligned}
$$

Relation (12) is equivalent to the following one :

$$
\left\{\begin{aligned}
\mathfrak{W}\left(f_{\alpha}^{e}, f_{\alpha}^{o}\right)(s)=\left[(1-\alpha) \sqrt{\mathfrak{W}\left(f_{1}^{e}, f_{1}^{o}\right)(s)}-\alpha \sqrt{\mathfrak{W}\left(f_{0}^{e}, f_{0}^{o}\right)(s)}\right]^{2}+ \\
2 \alpha(1-\alpha)\left[\sqrt{\mathfrak{W}\left(f_{0}^{e}, f_{0}^{o}\right)(s) \mathfrak{W}\left(f_{1}^{e}, f_{1}^{o}\right)(s)}+\frac{1}{2}\left(\mathfrak{W}\left(f_{0}^{e}, f_{1}^{o}\right)(s)+\mathfrak{W}\left(f_{1}^{e}, f_{0}^{o}\right)(s)\right)\right]
\end{aligned}\right.
$$

If $f_{\alpha}(s)$ is stable then we deduce from Theorem 2

1) Firstly, the roots of $f_{\alpha}^{e}\left(-s^{2}\right)$ are real for any $\alpha \in[0,1]$. Then, by considering Lemma 2 , we get for $k=1, \ldots, m-1$

$$
\max \left(s_{k}^{e_{0}}, s_{k}^{e_{1}}\right) \leq \min \left(s_{k+1}^{e_{0}}, s_{k+1}^{e_{1}}\right)
$$

2) Secondly, for any $s$ in $\mathbb{R}$ and for any $\alpha \in[0,1], \mathfrak{W}\left(f_{\alpha}^{e}, f_{\alpha}^{o}\right)(s)>0$. Then, we deduce that $\mathfrak{W}\left(f_{0}^{e}, f_{0}^{o}\right)(s)>0$ (case $\alpha=0$ ) and $\mathfrak{W}\left(f_{1}^{e}, f_{1}^{o}\right)(s)>0$ (case $\alpha=1$ ). The next step is widely inspired from proof of Lemma 2.1 in [1]. Let $\widetilde{\alpha}(s)$ defined by the following expression :

$$
\widetilde{\alpha}(s)=\frac{\sqrt{\mathfrak{W}\left(f_{1}^{e}, f_{1}^{o}\right)(s)}}{\sqrt{\mathfrak{W}\left(f_{1}^{e}, f_{1}^{o}\right)(s)}+\sqrt{\mathfrak{W}\left(f_{0}^{e}, f_{0}^{o}\right)(s)}}
$$

This yields to $\widetilde{\alpha}(s) \in(0,1)$. By taking $\alpha=\widetilde{\alpha}(s)$ for any $s \in \mathbb{R}$ in (13), this yields to

$$
\begin{aligned}
& \forall s \in \mathbb{R}, \\
& 2 \widetilde{\alpha}(s)(1-\widetilde{\alpha}(s))\left[\sqrt{\mathfrak{W}\left(f_{0}^{e}, f_{0}^{o}\right)(s) \mathfrak{W}\left(f_{1}^{e}, f_{1}^{o}\right)(s)}+\frac{1}{2}\left(\mathfrak{W}\left(f_{0}^{e}, f_{1}^{o}\right)(s)+\mathfrak{W}\left(f_{1}^{e}, f_{0}^{o}\right)(s)\right)\right]>0
\end{aligned}
$$

That implies

$$
\forall s \in \mathbb{R}, \quad \sqrt{\mathfrak{W}\left(f_{0}^{e}, f_{0}^{o}\right)(s) \mathfrak{W}\left(f_{1}^{e}, f_{1}^{o}\right)(s)}+\frac{1}{2}\left(\mathfrak{W}\left(f_{0}^{e}, f_{1}^{o}\right)(s)+\mathfrak{W}\left(f_{1}^{e}, f_{0}^{o}\right)(s)\right)>0
$$

Conversely, assume that conditions (11) holds. 
Then by considering Lemma 2, the roots of $f_{\alpha}^{e}\left(-s^{2}\right)$ are real thus by assumption the roots of $f_{0}^{e}\left(-s^{2}\right)$ and $f_{1}^{e}\left(-s^{2}\right)$ are real. Moreover, as relation (13) holds and if the following inequalities are satisfied

$$
\begin{aligned}
\forall s \in \mathbb{R}, \quad & \mathfrak{W}\left(f_{0}^{e}, f_{0}^{o}\right)(s)>0, \quad \mathfrak{W}\left(f_{1}^{e}, f_{1}^{o}\right)(s)>0, \\
& \sqrt{\mathfrak{W}\left(f_{0}^{e}, f_{0}^{o}\right)(s) \mathfrak{W}\left(f_{1}^{e}, f_{1}^{o}\right)(s)}+\frac{1}{2}\left(\mathfrak{W}\left(f_{0}^{e}, f_{1}^{o}\right)(s)+\mathfrak{W}\left(f_{1}^{e}, f_{0}^{o}\right)(s)\right)>0
\end{aligned}
$$

then for any $s \in \mathbb{R}, \mathfrak{W}\left(f_{\alpha}^{e}, f_{\alpha}^{o}\right)(s)>0$. Hence by Theorem $2, f_{\alpha}(s)$ is stable for all $\alpha \in[0,1]$.

From Theorem 4, let us examine now some particular cases.

A simplified form of Theorem 4 is presented in the following corollary when $f_{0}$ and $f_{1}$ are stable.

Corollary 2: Consider two stable polynomials $f_{0}$ and $f_{1}$ in $\mathbb{P}_{m}$ with leading coefficients of same sign and $f_{0}^{e}\left(-s^{2}\right)$ and $f_{1}^{e}\left(-s^{2}\right)$ two polynomials in $\mathbb{H}_{m}$ with their roots given by the sets (6) and (7). The polynomial segment $f_{\alpha}$ defined by (10) is stable iff the two relations below are verified.

$$
\left\{\begin{array}{l}
\forall s \in \mathbb{R}, \quad \sqrt{\mathfrak{W}\left(f_{0}^{e}, f_{0}^{o}\right)(s) \mathfrak{W}\left(f_{1}^{e}, f_{1}^{o}\right)(s)}+\frac{1}{2}\left(\mathfrak{W}\left(f_{0}^{e}, f_{1}^{o}\right)(s)+\mathfrak{W}\left(f_{1}^{e}, f_{0}^{o}\right)(s)\right)>0 \\
\forall k=1, \ldots, m-1, \quad \max \left(s_{k}^{e_{0}}, s_{k}^{e_{1}}\right) \leq \min \left(s_{k+1}^{e_{0}}, s_{k+1}^{e_{1}}\right)
\end{array}\right.
$$

Proof: Obvious, see Theorem 4.

Corollary 3: Consider two polynomials $f_{0}$ and $f_{1}$ in $\mathbb{P}_{m}$ with leading coefficients of same sign and with the same even part $f^{e}$. Then the polynomial segment $f_{\alpha}$ defined by (10) is stable iff $f_{0}$ and $f_{1}$ are stable.

Proof: It can be deduced from Theorem 2 than $f_{\alpha}(s)$ is stable for all $\alpha \in[0,1]$ if $f_{0}$ and $f_{1}$ are stable, thus

1) the polynomials $f_{0}$ and $f_{1}$ are stable. Then the roots of $f^{e}\left(-s^{2}\right)$ are real.

2) for any $s \in \mathbb{R}, \mathfrak{W}\left(f_{\alpha}^{e}, f_{\alpha}^{o}\right)(s)>0$. Hence by developing $\mathfrak{W}\left(f_{\alpha}^{e}, f_{\alpha}^{o}\right)(s)$, one get

$$
\mathfrak{W}\left(f_{\alpha}^{e}, f_{\alpha}^{o}\right)(s)=\alpha \mathfrak{W}\left(f^{e}, f_{0}^{o}\right)(s)+(1-\alpha) \mathfrak{W}\left(f^{e}, f_{1}^{o}\right)(s)
$$

As for any $s \in \mathbb{R}, \mathfrak{W}\left(f^{e}, f_{0}^{o}\right)(s)>0, \mathfrak{W}\left(f^{e}, f_{1}^{o}\right)(s)>0$, this implies $\mathfrak{W}\left(f_{\alpha}^{e}, f_{\alpha}^{o}\right)(s)>0$.

Conversely, if we have for all $s$ and for any $\alpha \in[0,1], \mathfrak{W}\left(f_{\alpha}^{e}, f_{\alpha}^{o}\right)(s)>0$, then for all $s \in \mathbb{R}$, $\mathfrak{W}\left(f^{e}, f_{0}^{o}\right)(s)>0, \mathfrak{W}\left(f^{e}, f_{1}^{o}\right)(s)>0$. Therefore $f_{0}$ and $f_{1}$ are stable.

Corollary 4: Consider two polynomials $f_{0}$ and $f_{1}$ in $\mathbb{P}_{m}$ with leading coefficients of same sign and with the same even part $f^{e}$. Then the polynomial segment $f_{\alpha}$ defined by (10) is stable iff the roots of $f^{e}\left(-s^{2}\right)$ are real and for any $s \in \mathbb{R}, \mathfrak{W}\left(f^{e}, f_{0}^{o}\right)(s)>0, \mathfrak{W}\left(f^{e}, f_{1}^{o}\right)(s)>0$.

Proof: Obvious, see Corollary 3 and Theorem 2.

The result given in Corollary 4 holds if the two endpoints have the same odd parts. 


\section{B. Stability of polynomial planes}

The next corollary is an extension of Corollary 4 and Lemma 3.1 in [7], in regards to a convex plane $f_{\alpha, \mu}$ built from convex combinations of the four real polynomials of same degree $f_{0}^{e}, f_{1}^{e}$, $f_{0}^{o}, f_{1}^{o}$ as given hereafter by relation (14).

$$
\forall(\alpha, \mu) \in[0,1]^{2}, f_{\alpha, \mu}(s)=(1-\alpha) f_{0}^{e}\left(s^{2}\right)+\alpha f_{1}^{e}\left(s^{2}\right)+(1-\mu) s f_{0}^{o}\left(s^{2}\right)+\mu s f_{1}^{o}\left(s^{2}\right)
$$

Corollary 5: Consider $f_{0}^{o}$, $f_{1}^{o}$ two polynomials in $\mathbb{P}_{m}$ and $f_{0}^{e}\left(-s^{2}\right)$ and $f_{1}^{e}\left(-s^{2}\right)$ two polynomials in $\mathbb{H}_{m}$ with the leading coefficients of $f_{0}^{e}, f_{1}^{e}$ of same sign and their roots defined by the sets (6) and (7). Then the convex plane $f_{\alpha, \mu}$ defined by (14) is stable iff conditions (15) hold

$$
\begin{cases}\forall k=1, \ldots, m-1, & \max \left(s_{k}^{e_{0}}, s_{k}^{e_{1}}\right) \leq \min \left(s_{k+1}^{e_{0}}, s_{k+1}^{e_{1}}\right), \\ \forall s \in \mathbb{R}, & \mathfrak{W}\left(f_{0}^{e}, f_{0}^{o}\right)(s)>0, \quad \mathfrak{W}\left(f_{0}^{e}, f_{1}^{o}\right)(s)>0 . \\ \mathfrak{W}\left(f_{1}^{e}, f_{0}^{o}\right)(s)>0, \quad \mathfrak{W}\left(f_{1}^{e}, f_{1}^{o}\right)(s)>0 .\end{cases}
$$

Proof: If $f_{\alpha, \mu}$ is stable then

1) the roots of the polynomial combination $(1-\alpha) f_{0}^{e}\left(-s^{2}\right)+\alpha f_{1}^{e}\left(-s^{2}\right)$ are real, then from Lemma 2 we have

$$
\forall k=1, \ldots, m-1, \quad \max \left(s_{k}^{e_{0}}, s_{k}^{e_{1}}\right) \leq \min \left(s_{k+1}^{e_{0}}, s_{k+1}^{e_{1}}\right)
$$

2) for any $s \in \mathbb{R}$, for any $\alpha \in[0,1]$, and for any $\mu \in[0,1], \mathfrak{W}\left(f_{\alpha}^{e}, f_{\mu}^{o}\right)(s)>0$ where

$$
\begin{aligned}
& f_{\alpha}^{e}\left(s^{2}\right)=(1-\alpha) f_{0}^{e}\left(s^{2}\right)+\alpha f_{1}^{e}\left(s^{2}\right) \\
& f_{\mu}^{o}\left(s^{2}\right)=(1-\mu) f_{0}^{o}\left(s^{2}\right)+\mu f_{1}^{o}\left(s^{2}\right)
\end{aligned}
$$

Therefore we have

$$
\left\{\begin{array}{c}
\forall \alpha \in[0,1], \forall \mu \in[0,1], \forall s \in \mathbb{R} \\
\mathfrak{W}\left(f_{\alpha}^{e}, f_{\mu}^{o}\right)(s)=(1-\alpha)(1-\mu) \mathfrak{W}\left(f_{0}^{e}, f_{0}^{o}\right)(s)+(1-\alpha) \mu \mathfrak{W}\left(f_{0}^{e}, f_{1}^{o}\right)(s) \\
+(1-\mu) \alpha \mathfrak{W}\left(f_{1}^{e}, f_{0}^{o}\right)(s)+\alpha \mu \mathfrak{W}\left(f_{1}^{e}, f_{1}^{o}\right)(s)>0
\end{array}\right.
$$

This implies that $\mathfrak{W}\left(f_{0}^{e}, f_{0}^{o}\right)(s)>0$ (case $\alpha=0, \mu=0$ ), $\mathfrak{W}\left(f_{0}^{e}, f_{1}^{o}\right)(s)>0$ (case $\alpha=0, \mu=1$ ), $\mathfrak{W}\left(f_{1}^{e}, f_{0}^{o}\right)(s)>0$ (case $\alpha=1, \mu=0$ ), $\mathfrak{W}\left(f_{1}^{e}, f_{1}^{o}\right)(s)>0$ (case $\alpha=1, \mu=1)$.

Conversely, if the roots of $f_{0}^{e}\left(-s^{2}\right)$ and $f_{1}^{e}\left(-s^{2}\right)$ are real and

$$
\forall k=1, \ldots, m-1, \quad \max \left(s_{k}^{e_{0}}, s_{k}^{e_{1}}\right) \leq \min \left(s_{k+1}^{e_{0}}, s_{k+1}^{e_{1}}\right)
$$

then the roots of the polynomial combination $(1-\alpha) f_{0}^{e}\left(-s^{2}\right)+\alpha f_{1}^{e}\left(-s^{2}\right)$ are real, see Lemma 2. Moreover

$$
\left\{\begin{array}{lll}
\forall s \in \mathbb{R}, & \mathfrak{W}\left(f_{0}^{e}, f_{0}^{o}\right)(s)>0, & \mathfrak{W}\left(f_{0}^{e}, f_{1}^{o}\right)(s)>0 \\
\mathfrak{W}\left(f_{1}^{e}, f_{0}^{o}\right)(s)>0, & \mathfrak{W}\left(f_{1}^{e}, f_{1}^{o}\right)(s)>0
\end{array}\right.
$$

then we have $\mathfrak{W}\left(f_{\alpha}^{e}, f_{\mu}^{o}\right)(s)>0$ for all $\alpha \in[0,1]$ and for all $\mu \in[0,1]$. Therefore we deduce from Theorem 2 that $f_{\alpha, \mu}$ is stable. 
Corollary 6: Consider four stable polynomials of degree $m, f_{00}, f_{01}, f_{10}, f_{11}$ such that

$$
\begin{array}{ll}
f_{00}(s)=f_{0}^{e}\left(s^{2}\right)+s f_{0}^{o}\left(s^{2}\right), & f_{10}(s)=f_{1}^{e}\left(s^{2}\right)+s f_{0}^{o}\left(s^{2}\right) \\
f_{01}(s)=f_{0}^{e}\left(s^{2}\right)+s f_{1}^{o}\left(s^{2}\right), & f_{11}(s)=f_{1}^{e}\left(s^{2}\right)+s f_{1}^{o}\left(s^{2}\right)
\end{array}
$$

where $f_{0}^{e}\left(-s^{2}\right)$ and $f_{1}^{e}\left(-s^{2}\right)$ are two polynomials in $\mathbb{H}_{m}$ with their roots given by the sets (6) and (7) and the leading coefficients of same sign. Then the convex plane of polynomials $f_{\alpha, \mu}$ defined by (14) is stable iff the following relation holds.

$$
\forall k=1, \ldots, m-1, \quad \max \left(s_{k}^{e_{0}}, s_{k}^{e_{1}}\right) \leq \min \left(s_{k+1}^{e_{0}}, s_{k+1}^{e_{1}}\right)
$$

Proof: The proof follows from Corollary 4 and Corollary 5.

The link between the stability of the four Kharitonov's polynomials and the stability of this Kharitonov's plane $f_{\alpha, \mu}$ is made by Corollary 5 and Corollary 6.

In the following theorem is produced a stability result for the polynomial combination $\alpha f_{0}(s)+$ $\mu f_{1}(s)$ with $(\alpha, \mu) \in \mathbb{R}^{2}$.

Theorem 5: Consider the polynomial combination $f_{\alpha, \mu}$ defined by (18)

$$
\forall(\alpha, \mu) \in \mathbb{R}^{2}, \quad f_{\alpha, \mu}(s)=\alpha f_{0}(s)+\mu f_{1}(s)
$$

where $f_{0}$ and $f_{1}$ are two polynomials in $\mathbb{P}_{m}$.

Denote by $f_{0}^{e}, f_{1}^{e}$ and $f_{0}^{o}$, $f_{1}^{o}$ respectively the even parts and the odd parts of $f_{0}$ and $f_{1}$. Assume that the roots of $f_{0}^{e}\left(-s^{2}\right)$ and $f_{1}^{e}\left(-s^{2}\right)$ verify the Zeroes Interlacing Property. Then the polynomial plane $f_{\alpha, \mu}$ defined by (18) is stable iff the relationships (19) hold.

$$
\begin{cases}\forall s \in \mathbb{R}, & \mathfrak{W}\left(f_{0}^{e}, f_{0}^{o}\right)(s)>0 \\ \forall s \in \mathbb{R}, & W\left(f_{0}^{e}\left(-s^{2}\right), f_{1}^{e}\left(-s^{2}\right)\right)(s) W\left(s f_{0}^{o}\left(-s^{2}\right), s f_{1}^{o}\left(-s^{2}\right)\right)(s)>0\end{cases}
$$

In order to prove Theorem 5, we need the following Obreschkoff's theorem given in [12].

Theorem 6: [12] Let $f_{0}^{e}\left(-s^{2}\right)$ and $f_{1}^{e}\left(-s^{2}\right)$ two real polynomials of degree differing by 1 at most. The two following statements are equivalent

i) the roots of $f_{0}^{e}\left(-s^{2}\right)$ and $f_{1}^{e}\left(-s^{2}\right)$ verify the Zeroes Interlacing Property.

ii) the polynomial combination $\alpha f_{0}^{e}\left(-s^{2}\right)+\mu f_{1}^{e}\left(-s^{2}\right)$ has only distinct real zeros for all reals $\alpha$ and $\mu$.

We are now in position to give a proof for Theorem 5 .

Proof of Theorem 5 : By considering (18), define $f_{\alpha, \mu}^{e}$ and $f_{\alpha, \mu}^{o}$ as the following expressions

$$
\begin{aligned}
& f_{\alpha, \mu}^{e}\left(s^{2}\right)=\alpha f_{0}^{e}\left(s^{2}\right)+\mu f_{1}^{e}\left(s^{2}\right) \\
& f_{\alpha, \mu}^{o}\left(s^{2}\right)=\alpha f_{0}^{o}\left(s^{2}\right)+\mu f_{1}^{o}\left(s^{2}\right)
\end{aligned}
$$

From Theorem 2, $f_{\alpha, \mu}$ is stable iff the two conditions a) and b) given hereafter hold.

a) Firstly, we can observe that $f_{\alpha, \mu}^{e}\left(-s^{2}\right)$ defined by (20) has only distinct real roots for any $(\alpha, \mu) \in \mathbb{R}^{2}$ since the roots of $f_{0}^{e}\left(-s^{2}\right)$ and $f_{1}^{e}\left(-s^{2}\right)$ verify the Zeroes Interlacing Property by assumption, see Theorem 6 . 
b) Secondly, for any $s \in \mathbb{R}$ and for any $(\alpha, \mu) \in \mathbb{R}^{2}$, we have $\mathfrak{W}\left(f_{\alpha, \mu}^{e}, f_{\alpha, \mu}^{o}\right)(s)>0$. Namely $\left\{\begin{array}{l}\forall(\alpha, \mu) \in \mathbb{R}^{2}, \forall s \in \mathbb{R}, \\ \mathfrak{W}\left(f_{\alpha, \mu}^{e}, f_{\alpha, \mu}^{o}\right)(s)=\alpha^{2} \mathfrak{W}\left(f_{0}^{e}, f_{0}^{o}\right)(s)+\alpha \mu \mathfrak{W}\left(f_{0}^{e}, f_{1}^{o}\right)(s)+\mu \alpha \mathfrak{W}\left(f_{1}^{e}, f_{0}^{o}\right)(s)+\mu^{2} \mathfrak{W}\left(f_{1}^{e}, f_{1}^{o}\right)(s)>0\end{array}\right.$

Relation (22) is equivalent to the following

$$
\begin{aligned}
\forall(\alpha, \mu) \in \mathbb{R}^{2}, \forall s \in \mathbb{R}, \\
{\left[\begin{array}{ll}
\alpha & \mu
\end{array}\right]\left[\begin{array}{ll}
\mathfrak{W}\left(f_{0}^{e}, f_{0}^{o}\right)(s) & \mathfrak{W}\left(f_{0}^{e}, f_{1}^{o}\right)(s) \\
\mathfrak{W}\left(f_{1}^{e}, f_{0}^{o}\right)(s) & \mathfrak{W}\left(f_{1}^{e}, f_{1}^{o}\right)(s)
\end{array}\right]\left[\begin{array}{l}
\alpha \\
\mu
\end{array}\right]>0 }
\end{aligned}
$$

Inequality (23) is satisfied iff the matrix $\mathbb{W}(s)$ hereafter is strictly positive definite for any real $s$.

$$
\mathbb{W}(s)=\left[\begin{array}{ll}
\mathfrak{W}\left(f_{0}^{e}, f_{0}^{o}\right)(s) & \mathfrak{W}\left(f_{0}^{e}, f_{1}^{o}\right)(s) \\
\mathfrak{W}\left(f_{1}^{e}, f_{0}^{o}\right)(s) & \mathfrak{W}\left(f_{1}^{e}, f_{1}^{o}\right)(s)
\end{array}\right]>0
$$

The matrix $\mathbb{W}(s)$ is strictly positive definite for any real $s$ iff all the determinants of the principal submatrices $\mathbb{W}(s)$ are strictly positive for any real $s$, i.e. iff we have $\mathfrak{W}\left(f_{0}^{e}, f_{0}^{o}\right)(s)>0$ and $|\mathbb{W}|>0$ for any real $s$. That is iff conditions (19) hold.

\section{CONCLUSION}

In this paper, results have been produced for studying the stability of real polynomials. For beginning Theorem 2 establishes an algebraic criterion of stability that may be summarized as a test of positiveness of only one univariate polynomial. In addition, Corollary 1 provides a tractable test for deciding of the polynomial stability. Finally, these results have been extended to analyze the stability of polynomial combinations in the case of convex segments of polynomials and in the case of planes of polynomials.

As the stability criteria given in this paper are mainly based on a polynomial positivity, this work can be profitably linked with a wide panel of developments produced these last years surrounding positive polynomials and sums of squares of polynomials, see [11].

\section{APPENDIX}

Proof of Theorem 2.

"if" : According to Theorem 1, it is sufficient to prove that

1) $f^{e}\left(-s^{2}\right)$ and $s f^{o}\left(-s^{2}\right)$ have simple and distinct roots,

2) the leading coefficients of $f^{e}\left(s^{2}\right)$ and $s f^{o}\left(s^{2}\right)$ have the same sign,

3 the roots of $s f^{o}\left(-s^{2}\right)$ are all real and interlace with the roots of $f^{e}\left(-s^{2}\right)$.

Let us show these three conditions.

1) If $s_{i}$ is a common zero of $f^{e}\left(-s^{2}\right)$ and $s f^{o}\left(-s^{2}\right)$ (or a common zero of $f^{e}\left(-s^{2}\right)$ and $\frac{d f^{e}\left(-s^{2}\right)}{d s}$ or a common zero of $s f^{o}\left(-s^{2}\right)$ and $\left.\frac{d f^{o}\left(-s^{2}\right)}{d s}\right)$ then $\mathfrak{W}\left(f^{e}, f^{o}\right)\left(s_{i+1}\right)=0$. 
2) Let us consider that $f$ is expressed by (1). The leading coefficient of $\mathfrak{W}\left(f^{e}, f^{o}\right)(s)$ is $\alpha_{m} \alpha_{m-1}$, where $\alpha_{m}$ and $\alpha_{m-1}$ are the leading coefficients of $f^{e}\left(s^{2}\right)$ and $f^{o}\left(s^{2}\right)$, respectively. Suppose that $\alpha_{m}$ and $\alpha_{m-1}$ have a different sign, then $\alpha_{m} \alpha_{m-1}$ is negative and so is $\mathfrak{W}\left(f^{e}, f^{o}\right)(s)$ for $s$ large enough.

3) Let us show that the zeroes of $f^{e}\left(-s^{2}\right)$ and $s f^{o}\left(-s^{2}\right)$ interlace. Let $s_{i}$ and $s_{i+1}$ be two negative and consecutive real zeroes of $f^{e}\left(-s^{2}\right)$. Then

$$
\begin{aligned}
\mathfrak{W}\left(f^{e}, f^{o}\right)\left(s_{i}\right) & =-\left.s_{i} f^{o}\left(-s_{i}^{2}\right) \frac{d f^{e}\left(-s^{2}\right)}{d s}\right|_{s=s_{i}}>0 \\
\mathfrak{W}\left(f^{e}, f^{o}\right)\left(s_{i+1}\right) & =-\left.s_{i+1} f^{o}\left(-s_{i+1}^{2}\right) \frac{d f^{e}\left(-s^{2}\right)}{d s}\right|_{s=s_{i+1}}>0
\end{aligned}
$$

Furthermore $\left.\frac{d f^{e}\left(-s^{2}\right)}{d s}\right|_{s=s_{i}}$ and $\left.\frac{d f^{e}\left(-s^{2}\right)}{d s}\right|_{s=s_{i+1}}$ have not the same sign . As the sign of $\mathfrak{W}\left(f^{e}, f^{o}\right)(s)$ is constant, then the sign of $s_{i} f^{o}\left(s_{i}\right)$ and $s_{i+1} f^{o}\left(s_{i+1}\right)$ are opposite. Therefore there is an odd number of real simple zeroes of $s f^{\circ}\left(-s^{2}\right)$ between $s_{i}$ and $s_{i+1}$.

From this statement we consider two cases :

i) $m$ is even, i.e the number of roots of $s f^{o}\left(-s^{2}\right)$ is one less than $f^{e}\left(-s^{2}\right)$. Therefore the roots of $s f^{o}\left(-s^{2}\right)$ are all real and interlace with the roots of $f^{e}\left(-s^{2}\right)$

ii) $m$ is odd, i.e. the number of roots of $s f^{o}\left(-s^{2}\right)$ is one more than $f^{e}\left(-s^{2}\right)$. Let us suppose that two roots of $s f^{o}\left(-s^{2}\right)$ are complex conjugate or that the roots of $s f^{o}\left(-s^{2}\right)$ and $f^{e}\left(-s^{2}\right)$ do not interlace. Then the greater root $s_{k}$ of $f^{e}\left(-s^{2}\right)$ is greater than the greater root of $s f^{o}\left(-s^{2}\right)$. This yields to

$$
\mathfrak{W}\left(f^{e}, f^{o}\right)\left(s_{k}\right)=-\left.s_{k} f^{o}\left(-s_{k}^{2}\right) \frac{d f^{e}\left(-s^{2}\right)}{d s}\right|_{s=s_{k}}>0
$$

Suppose that $\left.\frac{d f^{e}\left(-s^{2}\right)}{d s}\right|_{s=s_{k}}>0$ (the case $\left.\frac{d f^{e}\left(-s^{2}\right)}{d s}\right|_{s=s_{k}}<0$ is similar). Then $f^{e}\left(-s^{2}\right)$ is positive for $s>s_{k}$, and (25) implies that $f^{o}\left(-s^{2}\right)$ is negative for $s>s_{k}$, therefore the leading coefficients of $f^{e}\left(-s^{2}\right)$ and $f^{o}\left(-s^{2}\right)$ have opposite signs. This is in contradiction with 2$)$.

"only if" : We know from Theorem 1 that any root $s_{i}$ of $f^{e}\left(-s^{2}\right)$ is real and simple, and that the roots of $f^{e}\left(-s^{2}\right)$ and $s f^{o}\left(-s^{2}\right)$ interlace. Following [10], if $m=2 k$, then

$$
\frac{s f^{o}\left(-s^{2}\right)}{f^{e}\left(-s^{2}\right)}=\sum_{i=1}^{k} \frac{R_{i}}{s-s_{i}}
$$

where $s_{i}$ are the roots of $f^{e}\left(-s^{2}\right)$ and with

$$
R_{i}=\frac{s_{i} f^{o}\left(-s_{i}^{2}\right)}{\left.\frac{d\left(f^{e}\left(-s^{2}\right)\right)}{d s}\right|_{s=s_{i}}}=\frac{f^{o}\left(-s_{i}^{2}\right)}{-\left.2 \frac{d f^{e}(u)}{d u}\right|_{u=-s_{i}^{2}}}<0
$$

Indeed $\frac{f^{o}(u)}{\frac{d f^{e}(u)}{d u}}>0$ when $f^{e}\left(-s^{2}\right)$ and $s f^{o}\left(-s^{2}\right)$ interlace, see [10].

We obtain

$$
\frac{f^{e}\left(-s^{2}\right) \frac{d f^{o}\left(-s^{2}\right)}{d s}-s f^{o}\left(-s^{2}\right) \frac{d f^{e}\left(-s^{2}\right)}{d s}}{\left(f^{e}\left(-s^{2}\right)\right)^{2}}=-\sum_{i=1}^{k} \frac{R_{i}}{\left(s-s_{i}\right)^{2}}>0
$$


On the other side, if $m=2 k+1$ we have (from [10]) :

$$
\frac{s f^{o}\left(-s^{2}\right)}{f^{e}\left(-s^{2}\right)}=a_{0} s+b_{0}+\sum_{i=1}^{k} \frac{R_{i}}{s-s_{i}}
$$

where $a_{0}$ and $b_{0}$ are two constants. According to Theorem 1 the leading coefficients of $s f^{o}\left(s^{2}\right)$ and $f^{e}\left(s^{2}\right)$ have the same sign and as well those of $s f^{o}\left(-s^{2}\right)$ and $f^{e}\left(-s^{2}\right)$. This implies

$$
\frac{f^{e}\left(-s^{2}\right) \frac{d f^{o}\left(-s^{2}\right)}{d s}-s f^{o}\left(-s^{2}\right) \frac{d f^{e}\left(-s^{2}\right)}{d s}}{\left(f^{e}\left(-s^{2}\right)\right)^{2}}=a_{0}-\sum_{i=1}^{k} \frac{R_{i}}{\left(s-s_{i}\right)^{2}}>0
$$

\section{REFERENCES}

[1] L-E Anderson, G. Chang, and T. Elfving. Criteria for copositive matrices using simplices and barycentric coordinates. Linear Algebra and its Application, 220(9-30):pp 9-30, 1995.

[2] B.R Barmish. New tools for robustness of linear systems,. Mac Millan, 1994.

[3] S. P. Bhattacharyya, H. Chappellat, and L. H. Keel. Robust Control, The parametric Approach. Prentice Hall, 1995.

[4] S. Bialas. A necessary and sufficient condition for the stability of convex combinations of stable polynomials and matrices. Bulletin of Polish Academy, 33:473-480, 1985.

[5] V. Blondel. A note on convex combinations of polynomials. IEEE TAC, 41(11):pp. 1690-1691, 1996.

[6] N. K Bose. A system theoretic approach to stability of sets of polynomials. Contemporary Math, 47:pp 25-34, 1985.

[7] H. Chappellat and S. P. Bhattacharyya. An alternative proof of Kharitonov's theorem. IEEE-TAC, 34:pp. 448-450, 1989.

[8] J.P Dedieu. Obreschkoff's theorem revisited: What convex sets are contained in the set of hyperbolic polynomials ? Journal of Pure and Applied Algebra, 81:pp. 269-278, 1992.

[9] J.P Dedieu and R.J Gregorac. Corrigendum: Obreschkoff's theorem revisited: What convex sets are contained in the set of hyperbolic polynomials ? Journal of Pure and Applied Algebra, 93:pp. 111-112, 1994.

[10] F. R. Gantmacher. The Theory of Matrices. Chelsea Publishing Company, New York edition, 1959.

[11] D. Henrion and A. Garulli (Eds.). Positive Polynomials in Control. Macmillan Publishing Company, springer edition, 2005.

[12] N. Obreschkoff. Verteilung und Berechnung der Nullstellen reeller Polynome. VEB Deutscher Verlag der Wissenschaften, 1963.

[13] A. Olshevsky and V. Olshevsky. Kharitonov's theorem and bezoutians. Linear Algebra and its Applications, 399:pp. 285-297, 2005.

[14] L. Yang. Recent advances on determining the number of real roots of parametric polynomials. J. Symbolic Computation, 28:pp. 225-242, 1999.

[15] L. Yang, X.R. Hou, and Z.B Zeng. A complete discrimination system for polynomials. Sci China, E-39:pp. 628-646, 1996. 